\title{
Detección de Descargas Parciales en una Instalación Eléctrica Subterránea por Medio de Procesamiento Digital de Imágenes Térmicas
}

\section{Detection of Partial Discharges in an Underground Electrical Installation by means of Digital Thermal Image Processing}

Adrián González-Parada1, Rubén Jaramillo-Vacío², Ricard Bosch-Tous ${ }^{3}$, Pau Casals-Torrens ${ }^{4}$

González-Parada, A; Jaramillo-Vacío, R; Bosch-Tous, R; Casals-Torrens, P. Detección de descargas parciales en una instalación eléctrica subterránea por medio de procesamiento digital de imágenes térmicas. Tecnología en Marcha. Vol. 34, especial. ALTAE. Diciembre 2021. Pág 48-61.

do) https://doi.org/10.18845/tm.v34i7.6011

Universidad de Guanajuato. México. Correo electrónico: gonzaleza@ugto.mx

(iD https://orcid.org/0000-0003-3473-1349

2 LAPEM-CFE, México. Correo electrónico: ruben.jaramillo@cfe.mx (iD) https://orcid.org/0000-0002-5876-9643

3 Universidad Politécnica de Cataluña, España. Correo electrónico: ricard.bosch@upc.edu 


\section{Palabras clave}

Instalaciones eléctricas subterráneas; cables de energía; fallas en terminales, imágenes termográficas; proceso digital de imágenes.

\section{Resumen}

La instalación de cables de potencia se deben de considerar una serie de factores para asegurar un buen desempeño en su operación y así mejorar notablemente la confiabilidad del sistema donde se encuentra. Durante la instalación se ven involucrados el empleo de accesorios específicos para su conexión a sistemas aéreos o para conexión de equipos, tales accesorios son conocidos como terminales y empalmes. De acuerdo a las estadísticas estos accesorios presentan el 95\% de las fallas debido a problemas durante su intalación o vida útil, por lo que es necesario llevar a cabo estudios o monitoreos programados de la operación de las instalaciones eléctricoas con el fin de reducir riesgos y prevenir fallas en la instalación. En el presente trabajo se presenta un método de detección incipiente de falla, el cual se basa en el análisis de imagenes termográficas de los accesorios (empalmes y terminales) de media tensión en operación. El objetivo es identificar la zona de una terminal en la que exista una falla incipiente por descargas parciales, las cuales no se pueden observar con una imagen termográfica simple, sino que se requiere realizar un análisis y proceso digital de la imagen termografica a fin de identificar plenamente el punto de falla en donde con el procesamiento digital de imágenes es posible extraer información que a simple vista no se percibe en una imagen obtenida con una cámara termográfica. Se presentan los resultados obtenidos en laboratorio de fallas previamente establecidas en un modelo de instalación eléctrica subterránea.

\section{Keywords}

Underground electrical installations; power cables; terminal failures, thermographic images; digital imaging process.

\section{Abstract}

The installation of power cables must be considered a series of factors to ensure good performance in its operation and thus significantly improve the reliability of the system where it is located. During the installation, the use of specific accessories for connection to aerial systems or for connection of equipment is involved, such accessories are known as terminals and splices. According to the statistics, these accessories present $90 \%$ of the failures due to problems during their installation or its life, so it is necessary to carry out scheduled studies or monitoring of the operation of electrical installations in order to reduce risks and prevent installation failures. In the present work, an incipient fault detection method is presented, which is based on the analysis of thermographic images of the accessories (splices and terminals) of medium voltage in operation. The objective is to identify the area of a terminal in which there is an incipient failure due to partial discharges, which cannot be observed with a simple thermographic image, but rather requires a digital analysis and processing of the thermographic image in order to identify the point of failure where with digital image processing it is possible to extract information that is not perceived by the naked eye in an image obtained with a thermal imaging camera. The results obtained in the laboratory of faults previously established in an underground electrical installation model are presented. 


\section{Introducción}

Un cable de potencia cumple la función de transportar energía eléctrica en media tensión o alta tensión, en instalaciones eléctrica subterránea, este tipo de instalaciones para llevarse a cabo se hace por medio de empalmes, que sirven para hacer secciones longitudinales mas largas cuando el tramo de la sección del cable no alcanza a cubrir la longitud total y de terminales las cuales son accesorios para hacer la transición aérea a la subterránea, en donde de acuerdo a las estadísticas de falla de este tipo de instalaciones eléctricas se presentan principalmente en los empalmes y las terminales; debido a que son elementos externos a la fabricación del cable. En la figura 1 se muestra los porcentajes de falla en una instalación eléctrica subterránea.

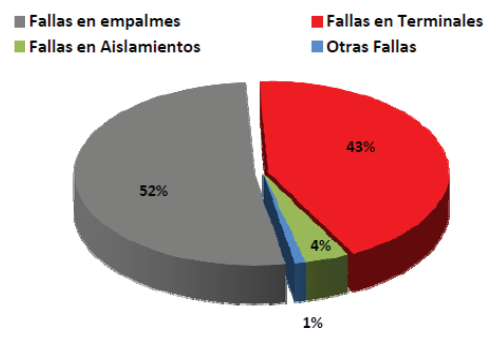

Figura 1 Porcentajes de falla en una instalación eléctrica subterránea.

De acuerdo a la figura 1, el mayor porcentaje se presenta en los empalmes que se instalan en los cables de media y alta tensión con un $52 \%$, seguido de las terminales con un 43\%, esto debido principalmente a que son elementos externos a la instalación y muchas de las veces no se tienen en cuenta los requerimientos necesarios para su instalación. En algunos casos, estas acciones han tenido consecuencias desastrosas ejemplo de ello son los incendios ocurridos en el centro histórico de la Ciudad de México debido a fallas en instalaciones eléctricas subterráneas, otro caso documentado es la anomalía en la terminal de la fase B, dentro del gabinete de los transformadores de medición en un edificio de un conocido banco en la ciudad de Querétaro, México, es necesario mencionar que tal anomalía no causó salida de línea porque gracias a una oportuna inspección visual se detectó la ruptura del cuerpo de la terminal. Sin embargo, sí fue necesario programar la posterior interrupción del suministro eléctrico para realizar el reemplazo de la terminal dañada. La figura 2 muestra la ruptura del cuerpo de la terminal. En el análisis de la falla se hallaron defectos de corte de la capa semiconductora, los cuales causaron una grieta que se extendió por la fuerza de contracción de la terminal. Además, esta preparación carece de un componente que el fabricante proporciona junto con la terminal que es la masilla graduadora de campo eléctrico [1],[2].

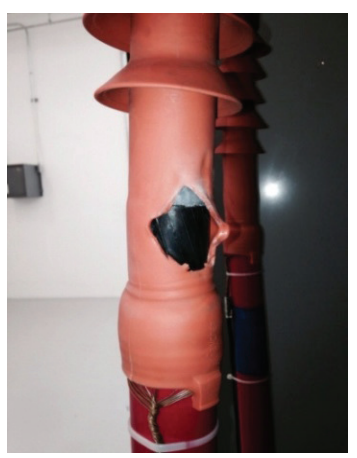

Figura 2. Ruptura de la cubierta de la terminal antes de una falla catastrófica. 
Debido a estas características de las instalaciones muchas de las veces se hacen inspecciones periódicas para verificar el estado en que se encuentra la instalación siendo la mayoría de las veces visuales y en algunos casos se emplea la técnica de la inspección visual por medio de la técnica de la termografía infrarroja.

La termografía infrarroja es una técnica que permite medir temperaturas a distancia y sin necesidad de contacto físico con el objeto a estudiar, mediante la captación de la intensidad de radiación infrarroja que emiten los cuerpos. Utilizando cámaras termográficas podemos convertir la energía radiada, invisible al ojo humano, en una imagen visible formada a partir de la temperatura superficial de los objetos captados por la cámara. Las cámaras termográficas actúan como un escáner termográfico sobre un objeto. La imagen captada por la cámara muestra en cada uno de sus puntos información de la temperatura del objeto. La cámara termográfica puede medir tanto un punto concreto como darnos una visión de la temperatura en diferentes zonas de un área determinada. Es decir, las cámaras termográficas basadas en infrarrojos nos permiten no sólo medir un punto sino la temperatura de todos los puntos captados por la imagen. De acuerdo con la tecnología actual de las cámaras termográficas muchas de la veces no es posible realizar y conocer directamente la zona directa de falla a menos que la diferencia de temperatura sea muy marcada, lo que para fines de inspección resulta impráctico, debido a que para poder detectar la falla tendría que ser muy alta y cuando se pudiera detectar esto sería demasiado tarde ya que la falla sería muy evidente [3],[4].

En el presente trabajo analizaremos los mecanismos de falla que se presentan en las instalaciones eléctricas subterráneas y la metodología de detección de falla por medio de la técnica termografía infrarroja y el proceso de digitalización de la imagen para detectar el punto de falla, para lo cual se hace necesario el conocimiento de los diferentes componentes de la una instalación eléctrica de media tensión y los mecanismos de falla involucrados y a partir de este conocimiento se propone la metodología para el empleo del análisis digital de imágenes a partir de termografía infrarroja.

\section{Cable de energía subterráneo}

Los cables de energía subterráneos para media y alta tensión son fabricados con diferentes componentes dieléctricos de tal manera que esta combinación puede ser un punto de falla, a continuación, en la figura 3, se presenta un esquema de los diferentes elementos que forman un cable típico para instalaciones eléctricas subterráneas.

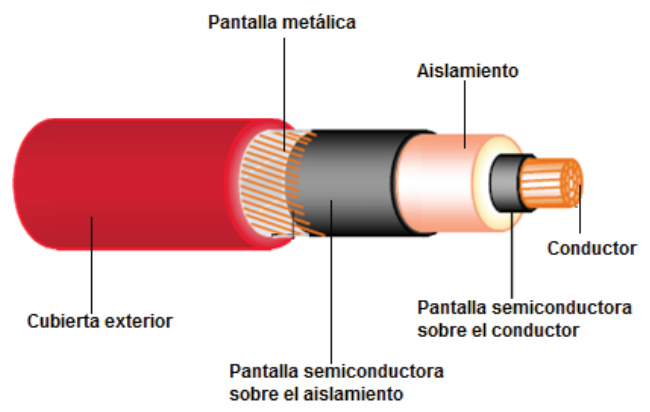

Figura 3. Esquema de un cable de energía subterráneo y sus componentes principales [5].

Cada uno de estos se describen a continuación de acuerdo a la operación dentro del sistema dieléctrico. 
1. Conductor.- Elemento que transporta la corriente del sistema y se encuentra al nivel de tensión de operación del cable, este puede ser de Cu o Al.

2. Pantalla semiconductora sobre el conductor.- Como transición entre el conductor y el aislamiento, con esta se cubren los intersticios presentes entre cada uno de los hilos externos que conforman al conductor, para evitar concentraciones de esfuerzos de campo eléctrico sobre el aislamiento, al presentar una superficie equipotencial uniforme.

3. Aislamiento.- Barrera dieléctrica entre el conductor y el punto de tierra mas próximo; los dieléctricos mas usados son el Etileno Polipropileno (EP) y el Polietileno de cadena cruzada $(X L P)$, siendo este último el mas usado por la industria eléctrica por su resistencia a las descargas parciales.

4. Pantalla semiconductora sobre aislamiento.- Esta tiene la función de uniformizar el campo eléctrico presente sobre el aislamiento y confinarlo en el interior del cable y su propósito específico es evitar que entre la pantalla metálica y el aislamiento quede una capa de aire ionizable con zonas de alta concentración de campo eléctrico alrededor del aislamiento.

5. Pantalla metálica.- Puede estar constituida por alambres o cintas ya sea de Cu o Al, siendo su función principal el proporcionar la seguridad en la instalación al estar conectada a un punto de tierra, ya que esta conduce la corriente de falla a tierra y proporciona protección a los operarios contra choque eléctrico.

6. Cubierta exterior.- Es la protección externa del cable contra elementos ambientales y daños mecánicos y esta construida con Cloruro de Polivinilo (PVC).

\section{Accesorios para cables de energía subterráneos}

\section{Terminales}

Las terminales son un accesorio que sirve para hacer la transición de una línea aérea a la línea subterránea; para poder llevar a cabo esta transición es necesario remover completamente las pantallas sobre el conductor y sobre el aislamiento y sustituir éstas por un sistema graduador de los esfuerzos eléctricos que se presentan con el fin de evitar concentraciones de campo que puedan dañar el material aislante y provocar una falla del sistema. Este al ser un sistema dielécrico ajeno al cable representa un punto de falla muy importante en la instalación cuando no se realiza de manera adecuada.

\section{Empalmes}

El empalme es unión de dos secciones de cable entre sí y se puede visualizar mejor como dos terminales conectadas entre sí, además de la conexión y reconstrucción de todos los elementos que constituyen el cable de potencia aislado, protegidos mecánicamente dentro de una misma cubierta. La diferencia más importante, desde un punto de vista teórico, entre los empalmes y las terminales es que los empalmes son más casi extensiones del cable. El empalme simplemente sustituye todos los componentes que se colocaron de fábrica en un cable por componentes de campo.

Ambos extremos del cable se preparan de la misma manera. En cada extremo del empalme donde se ha desprovisto del componente de blindaje del cable, se requiere un alivio de esfuerzos eléctricos tal como estaba en la estructura original del cable. La confiabilidad de un empalme para cables con aislamiento extruido depende de varios factores, entre los que destacan: la calidad de los materiales empleados, el diseño y la mano de obra de instalación. 


\section{Mecanismo de falla en dieléctricos sólidos}

Existen diversos mecanismos de falla en dieléctricos sólidos y estos tienen que ver principalmente con la velocidad del incremento de la tensión aplicada, la duración de aplicación de estas y las condiciones ambientales. En el caso de las instalaciones eléctricas subterráneas de media y alta tensión la principal causa de falla es la debida a las descargas parciales que se presentan debida a la concentración del campo eléctrico en puntos específicos de los accesorios. A continuación se describe este mecanismo de falla y sus efectos en el dieléctrico.

\section{Descargas parciales e incremento de temperatura}

Este tipo de rompimiento se presenta cuando existe diferentes materiales los cuales están expuesto a un mismo nivel de tensión; esto hace que se presenten concentraciones de campo eléctrico en el material con el valor de permitividad mas bajo. Si uno de los materiales es un gas o un líquido, entonces la tensión de ruptura medido será influenciado más por el medio débil o con permitividad mas baja, esto se muestra en la figura 4, en una configuración esfera-plano quedando un espacio que provoca descargas superficiales en los brodes del eleéctrodo [6-8].

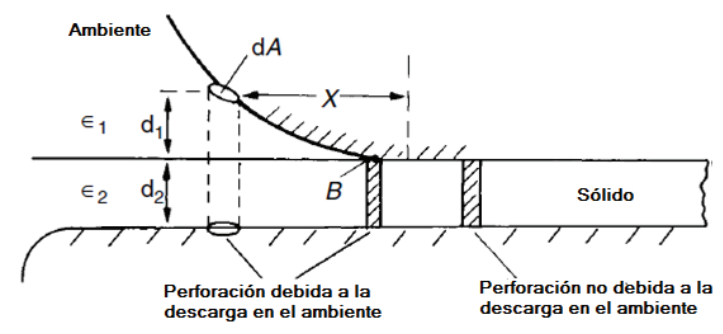

Figura 4. Rompimiento superficial en una configuración esfera-plano [6].

Si consideramos un volumen cilíndrico diferencial con área $d A$ abarcando los electrodos a distancia $x$ como muestra la figura 4, entonces al aplicar una tensión $V$ entre los electrodos, aparece una fracción $V_{1}$ del tensión a través del ambiente dada por la ecuación 1[9-11].

$$
V_{1}=\frac{V d_{1}}{d_{1}+\left(\frac{\varepsilon_{1}}{\varepsilon_{2}}\right) d_{2}}
$$

Donde, $d_{1}$ y $d_{2}$ representan los espesores de cada uno de los dieléctricos y $\varepsilon_{1}$ y $\varepsilon_{2}$ sus permitividades respectivas. El esfuerzo en la parte gaseosa excederá la del sólido por la relación de permitividades o $E_{1}=\varepsilon_{\mathrm{r}} E_{2}$. Para el caso mostrado en la figura 4 el esfuerzo en la parte gaseosa se incrementa a medida que la distancia al electrodo $x$ disminuye, y alcanza muy altos valores a medida que $d_{1}$ se hace muy pequeño (punto B). Esto produce una generación de calor debido a corrientes de polarización y decargas superficiales e internas, lo que nos lleva al análisis del rompimiento térmico.

\section{Rompimiento térmico}

Cuando un aislante está sometido a esfuerzo debido a las corrientes de conducción y pérdidas dieléctricas por polarización, dentro del dieléctrico se genera calor continuamente, dicho calor se transfiere a través del aislante y también una parte es radiada hacia las superficies. En general, la conductividad $(\sigma)$ incrementa con la temperatura, las condiciones de inestabilidad se alcanzan cuando la razón de calentamiento excede la razón de enfriamiento y entonces la muestra puede sufrir rompimiento térmico. 
Existen dos casos para el cálculo del rompimiento térmico. El primero asume un rápido aumento de calor, así que las pérdidas de calor hacia alrededores del material dieléctrico pueden ser despreciadas y todo el calor generado es usado en la elevación de la temperatura del sólido. Uno supone que surge una condición crítica y se pierden las propiedades del aislante cuando en algún punto en el dieléctrico la temperatura excede una temperatura crítica $T_{c}$. La ecuación para el cálculo de la situación crítica térmica es la siguiente [7].

$$
E_{c}=\sqrt{\frac{3 C_{v} k T_{0}^{2}}{\sigma_{0} u T_{c}}} \exp \left(\frac{u}{2 k T_{0}}\right)
$$

Donde, $C_{v}$ es la capacidad térmica del dieléctrico, $T_{c}$ es la temperatura crítica, $T_{0}$ es la temperatura ambiente, $\sigma_{0}$ es la conductividad eléctrica a temperatura ambiente, $k$ es la constante de conductividad térmica del dieléctrico, A y u son constantes empíricas dependientes del material.

Otro caso dentro de este tipo de rompimiento se refiere a la tensión térmica mínima, es decir, la menor tensión eléctrica para rompimiento térmico. Para este caso suponemos una placa dieléctrica, que está limitada a temperatura ambiente en sus superficies usando electrodos suficientemente grandes.

Al aplicar la tensión eléctrica, después de cierto tiempo, se establecerá una distribución de la temperatura dentro del dieléctrico, con la temperatura más alta en el centro $\left(T_{1}\right)$ que en la superficie, la cual permanecerá a temperatura ambiente. Al aumentar el nivel de tensión a un nuevo valor más alto, se establecerá un equilibrio a una temperatura central más alta $\left(T_{2}\right)$. Si se continúa el proceso, se producirá un rompimiento térmico como se muestra en la figura 5.

La ecuación para el cálculo de la tensión crítica de la ruptura térmica, donde $T_{c}$ es la temperatura crítica a la cual el material se descompone y para el cálculo se asume que $T_{c}$ corresponde al centro de la muestra, se da a continuación

$$
V_{c}^{2}=8 \frac{k}{\sigma_{0}}\left[\int_{T_{0}}^{T_{c}} \exp \left(\frac{u}{k T}\right) d T\right]
$$

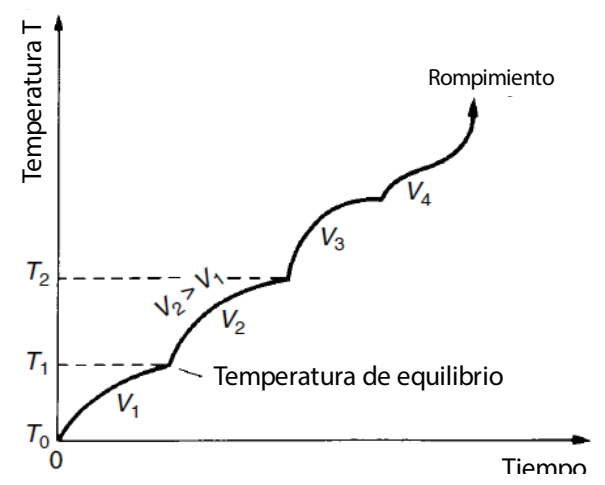

Figura 5. Rompimiento térmico en un material dieléctrico [6].

En tensiones de corriente alterna las pérdidas son mucho más grandes que en corriente directa. En consecuencia, el esfuerzo de rompimiento térmico es generalmente menor para corriente alterna y disminuye con el incremento de la frecuencia de la fuente de tensión. El rompimiento térmico es un mecanismo bien establecido, por lo tanto la magnitud del producto $\varepsilon t a n \delta$ el 
cual representa las pérdidas es un parámetro muy esencial para la aplicación de materiales aislantes. Al aplicar una tensión alterna, las pérdidas de potencia real $\left(P_{\mathrm{ac}}\right)$ un volumen $V$ de dieléctrico en un campo uniforme $E$ viene dada por la siguiente ecuación [7],[8].

$$
P_{a c}=\varepsilon_{0} \omega E^{2} V \varepsilon_{r} \tan \delta
$$

El término $\varepsilon_{r} \tan \delta$, es definido como "índice de pérdidas", dependiendo de la temperatura del dieléctrico, la frecuencia de alimentación y magnitud de la tensión aplicada. Para estimar la cantidad de energía producida, también se debe tener en cuenta la duración de aplicación de la tensión.

\section{Evaluación termográfica infrarroja}

Actualmente existen tecnologías desarrolladas con ciertos objetivos, a los cuales podemos sumar algún otro con algo de creatividad. Tal es el caso de las cámaras termográficas, cuyo propósito principal es el de medición de temperatura. Mezclando varias tecnologías es posible llegar a resultados más concluyentes en lo que respecta a un diagnóstico en particular. Para la finalidad buscada en este trabajo, la herramienta adicional será el procesamiento digital de imágenes y con ello llevará a cabo el cambio de aspecto de imágenes termográficas.

La fuente primaria de la radiación infrarroja es el calor o radiación térmica. Cualquier objeto que tenga una temperatura superior al cero absoluto irradia ondas en la banda infrarroja. Incluso los objetos que consideramos muy fríos emiten en el infrarrojo. Cuando un objeto no es suficientemente caliente para irradiar ondas en el espectro visible, emite la mayoría de su energía como ondas infrarrojas. Mientras más caliente se encuentre un objeto, mayor será la radiación infrarroja emitida [12].

\section{Procesamiento digital de imágenes}

El procesamiento de imágenes tiene como objetivo mejorar el aspecto de las imágenes o hacer más evidentes en ellas ciertos detalles que se desean hacer notar. La imagen puede haber sido generada de muchas maneras, por ejemplo, fotográficamente, o electrónicamente. El procesamiento de las imágenes se puede en general hacer por medio de métodos ópticos, o bien por medio de métodos digitales, en una computadora.

A grandes rasgos, el teorema de Fourier afirma que una gráfica o función, cualquiera que sea su forma, se puede representar con alta precisión dentro de un intervalo dado, mediante la suma de una gran cantidad de funciones senoidales, con diferentes frecuencias. Dicho de otro modo, cualquier función, sea o no sea periódica, se puede representar por una superposición de funciones periódicas con diferentes frecuencias.

La variación de la irradiancia o brillantez de una imagen, medida a lo largo de una dirección cualquiera es entonces una función que se puede representar mediante el teorema de Fourier, con una suma de distribuciones senoidales de varias frecuencias. Aquí es posible afirmar que atenuar o reforzar individualmente algunas de estas componentes senoidales puede tener un efecto dramático en la calidad de una imagen. Este es el fundamento del procesamiento de imágenes, tanto por medios ópticos como digitales [13],[14].

El procesamiento digital de imágenes se efectúa dividiendo la imagen en un arreglo rectangular de elementos. Cada elemento de la imagen así dividida se conoce con el nombre de pixel. El siguiente paso es asignar un valor numérico a la luminosidad promedio de cada pixel. Así, los valores de la luminosidad de cada pixel, con sus coordenadas que indican su posición, definen completamente la imagen. Todos estos números se almacenan en la memoria de una computadora [15],[16]. 
El tercer paso es alterar los valores de la luminosidad de los pixeles, a fin de hacer que resalten los detalles de la imagen que sean convenientes. En la figura 6, se presenta el diagrama de flujo del proceso de digitalización de la imagen termográfica.

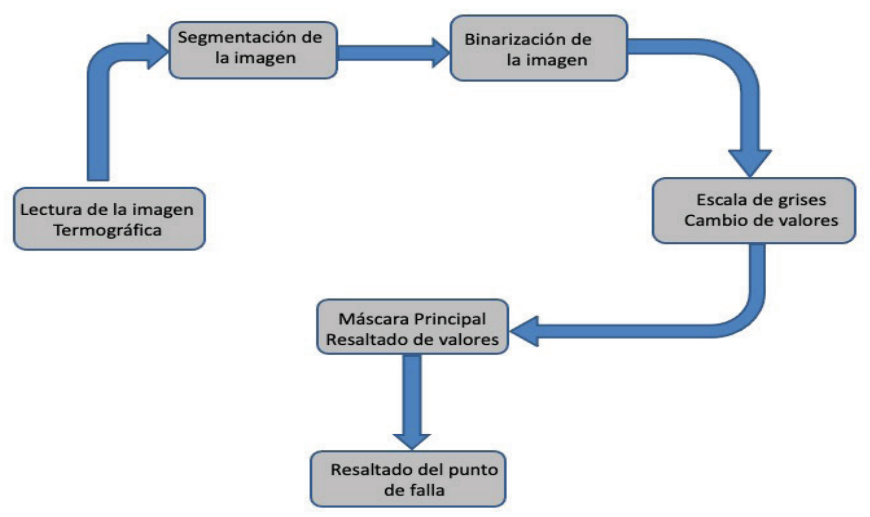

Figura 6. Proceso de digitalización de la imagén termográfica.

Aunque en algunos artículos publicados [17] ya ha sido tratado el tema de procesamiento digital de imágenes para detección de fallas en terminales de media tensión, el resultado final planteado en este trabajo es distinto. Aquí se pretende resaltar el punto de falla en una terminal de media tensión manteniendo visible el cuerpo de la terminal, para poder indentificar plenamente el punto de falla.

\section{Materiales y metodología}

\section{Implementación en laboratorio}

El sistema de prueba consiste en el conjunto de elementos que simulan en laboratorio un circuito de media tensión, cuya operación es similar a la de una transición aéreo-subterráneo. En los extremos de un cable de energía están colocadas dos terminales de silicón de la marca Elastimold, modelo PCT1, una de las cuales está en buenas condiciones mientras que la opuesta tiene un defecto causado intencionalmente (figura 7), de ésta manera se pretende caracterizar el patrón de falla para comparar y distinguir el comportamiento de ambos casos. Se empleo un cable de media tensión de $15 \mathrm{kV}$, conductor de Al, calibre 1/0 AWG cuerda compacta, aislamiento de XLP, 100\% nivel de aislamiento. Los extremos del cable son conectados a través de un conductor para formar un circuito cerrado para la circulación de corriente.

Las terminales y el cable están diseñados para funcionar con una tensión máxima de 15 kV, si se exceden los valores nominales de operación habrá daño en los componentes del sistema provocado por valores excesivos. 


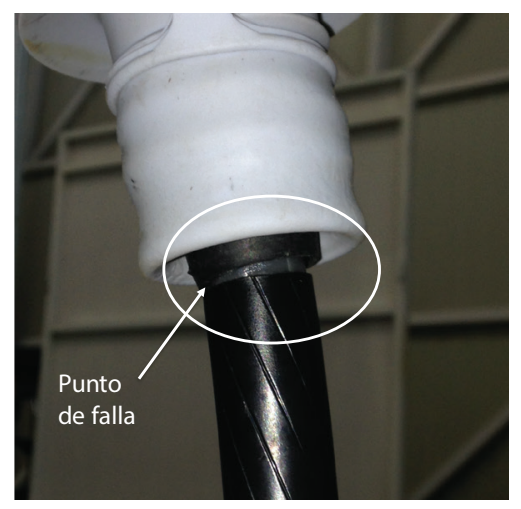

Figura 7. Terminal de $15 \mathrm{kV}$ con defecto en la instalación.

La alimentación eléctrica del circuito se lleva a cabo de la siguiente manera: Se aplicó una tensión de $15 \mathrm{kV}$ al conductor del cable mediante un transformador de potencial, éste es a su vez alimentado en su lado de baja tensión por una fuente variable de 0-110 V de tensión monofásica. Simultáneamente, al cable se le aplica una intensidad de corriente de 60 A con la ayuda de un transformador de corriente del tipo toroidal, mismo que se alimenta en sus terminales con una fuente de corriente variable 0-110 V, aplicando el valor de corriente nominal al cable. Se obtiene así la operación idéntica a la de una instalación eléctrica en campo, al presentar la tensión de operación y la corriente de carga del sistema. El esquema del circuito de prueba es representado en la figura 8 indicando la posición de cada componente.

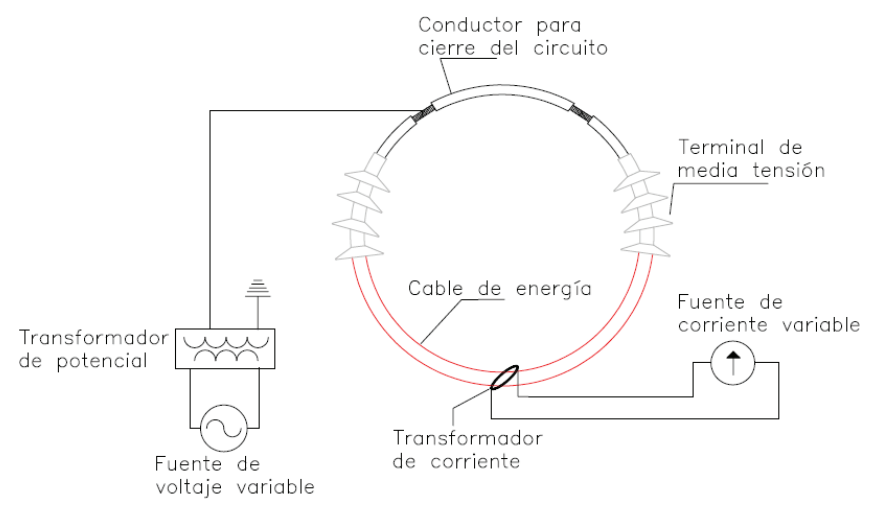

Figura 8. Esquema del sistema de prueba.

Para monitorear el comportamiento del cable y la presencia de las descargas parciales, se colocaron sensores del tipo inductivo bajo el cuerpo de la terminal en cada extremo de la instalación, esto permite detectar la actividad de las descargas en la terminal con falla y en la terminal sin falla. En la figura 9 se muestra la colocación del sensor en la terminal con falla, siendo similar a la colocación en el extremo sin falla. 


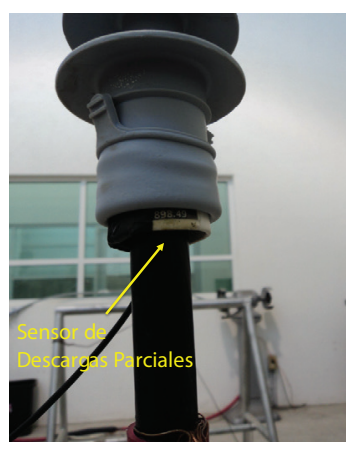

Figura 9. Colocación del sensor en el cuerpo de la terminal con falla.

Para la adquisición de la información obtenida con el sensor, éste es conectado a un osciloscopio (Fluke 190-102) mediante un cable coaxial. Las gráficas obtenidas con el osciloscopio se muestran en la figura 10.

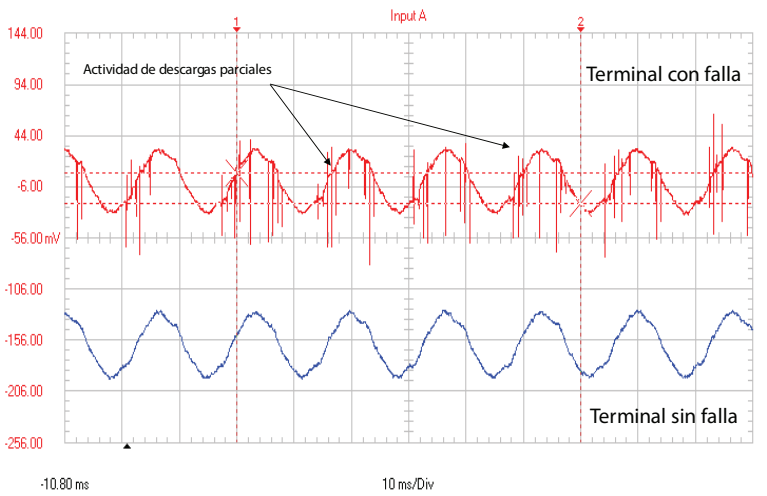

Figura 10. Comportamiento de las terminales con defecto y sin defecto en la instalación.

En donde se observa al actividad de las descargas en la terminal con falla y en el extremo contrario no se observa las descargas parciales. Esta diferencia nos da el patrón en el método eléctrico para distinguir el buen o mal estado de una terminal de media tensión.

\section{Detección por Termografía Infrarroja y digitalización de la imagen}

Se dejo el circuito operando en las condiciones de la sección 2.1 por cierto tiempo con el objetivo de que estabilizara el sistema y posteriormente se tomaron fotografias con cámara termografica FLUKE Ti480, en donde la imagen infrarroja de cada una de las terminales es de manera similar.

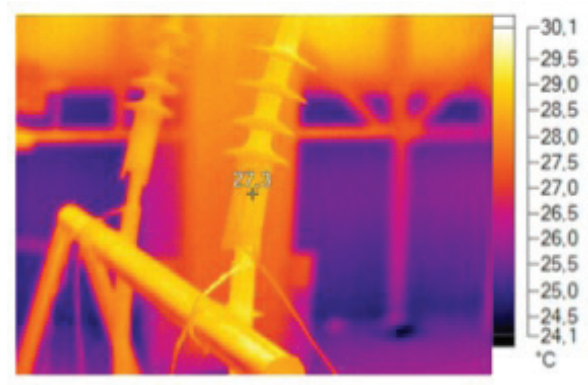

(a)

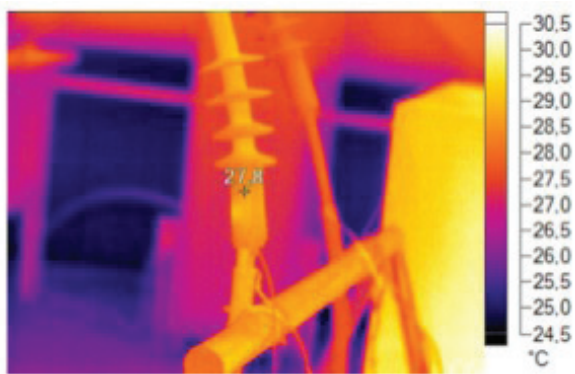

(b)

Figura 11. Imágenes termográficas infrarrojas de las terminales del circuito de prueba. a) terminal sin falla; b) terminal con falla. 
De la figura 11 se observa que la imagen térmica infrarroja obtenida de ambas terminales, no se pude distinguir el comportamiento de la terminal con falla a pesar de que esta presenta actividad de descargas parciales, por lo que esta simple evaluación no puede ser implementada directamente a la detección de fallas en una instalación eléctrica de este tipo.

Ya que la termografía consiste en la captación de radiación infrarroja, con el algoritmo se pretende aislar el rojo en distintos tonos pasando por varias etapas, en donde el procedimiento desarrollado es el siguiente:

1. Se lleva a cabo la lectura y almacenamiento de la imagen obtenida con la cámara termográfica.

2. La imagen se convierte a escala de grises.

3. La imagen es separada en sus bandas RGB. La banda roja es de principal interés para este trabajo puesto que el infrarrojo es, dentro del espectro electromagnético, el intervalo de medición de temperatura por medios termográficos. El hecho de trabajar con la banda roja en escala de grises nos permitirá más fácilmente establecer niveles de tonalidades posteriormente. El procesamiento consiste en identificar niveles de rojo de acuerdo a una temperatura de interés.

4. Se busca determinar automáticamente las zonas predominantemente rojas, es decir, las zonas "más brillantes" en la escala de grises de la banda roja.

5. Por medio de la función de corrección gamma se aumenta la luminosidad de la imagen obtenida en el paso anterior para resaltar las zonas rojas. Aquí se selecciona con el puntero del mouse el punto de sospecha de falla.

6. Se binariza la imagen, las zonas con un nivel elegido de rojo quedan en blanco y lo que se encuentre debajo de tal nivel queda en negro.

7. Por último, se resalta el color rojo en la zona elegida previamente con el puntero. Esto se logra asignando un valor de cero a los pixeles de las bandas verde y azul.

La figura 12 ilustra gráficamente los pasos seguidos para obtener el resultado buscado
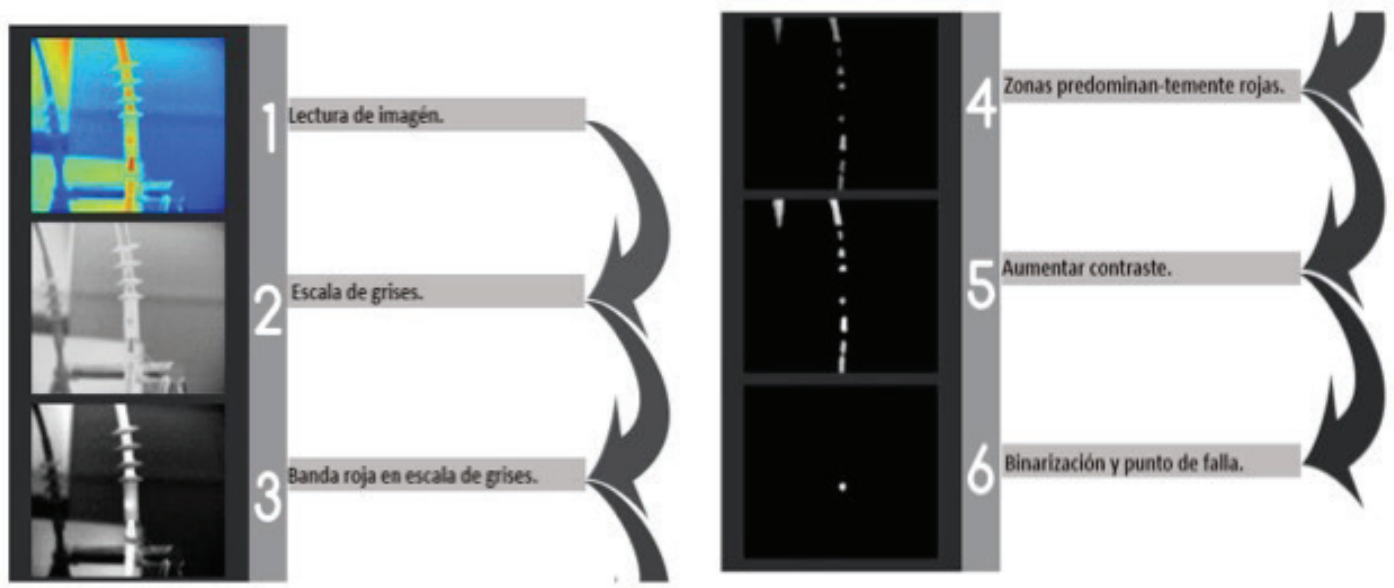

Figura 12. Proceso de digitalización de la imagen termogáfica. 


\section{Resultados}

La combinación de la termografía y el procesamiento digital de imágenes brinda la ventaja de que además de ser un método no intrusivo de detección de falla, abarca una mayor superficie para la realización del análisis en el que se persigue conocer si se presenta una concentración de calor que indique alguna irregularidad en la operación de la terminal debido a una falla incipiente, provocada por la actividad eléctrica de las descargas parciales.

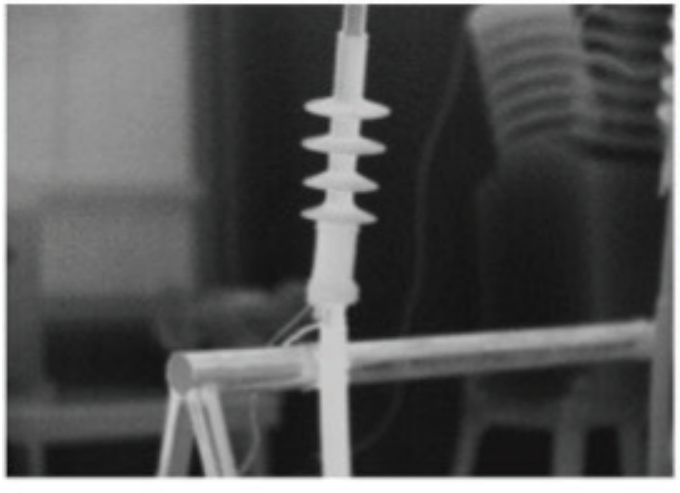

(a)

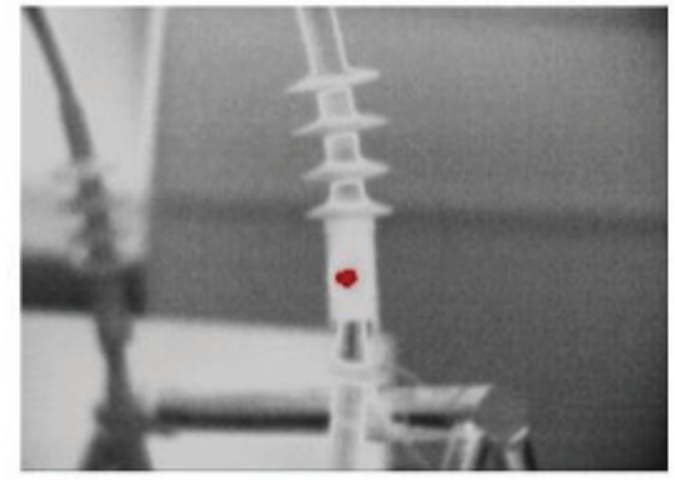

(b)

Figura 13. Procesamiento digital de las imágenes térmicas infrarrojas. (a) Terminal sin falla. (b) Terminal con falla.

La figura 13 muestra los resultados de la imagen procesada de la configuración experimental. Se puede ver claramente que la región del punto de falla (figura 13b) puede ser identificada con éxito en comparación de la figura de la terminal sin falla (figura 13a), en donde no se aprecia ninguna variación en a la textura y color.

En el análisis termográfico se requiere experiencia en la caracterización de este tipo de fallas, es decir, una vez que se sabe cómo se presenta una concentración de calor debida a descargas parciales, será más sencillo discernir para otro caso si lo observado es consecuencia de una falla en el sistema aislante. Es necesario que no interfieran otras fuentes reflejantes de luz ya que ello puede causar variación en los efectos térmicos causados por las descargas parciales. A pesar de esto, se puede lograr una medición precisa de la temperatura sin contacto con una buena calibración de la cámara termográfica.

\section{Conclusiones y/o recomendaciones}

El procesamiento propuesto en este trabajo cumplió con el objetivo primordial: desarrollar mejoras dirigidas a la localización de descargas parciales en terminales para cables de energía por medio del procesamiento digital de imagnes. Para ello se llevó a cabo la implementación de herramientas teóricas, experimentales y computacionales que condujeron a la caracterización de fallas eléctricas de manera indirecta, es decir, sin medición de parámetros eléctricos, sino con la medición de temperatura. Un punto importante a recalcar es que los resultados obtenidos presentan mejoras respecto a trabajos publicados [17].

Las nuevas tecnologías en terminales para cables de energía se han enfocado en una instalación sencilla, motivo por el cual los instaladores pueden llegar a abusar de la autoconfianza para la instalación de dichos componentes, la cual no permite error alguno (como suciedad o malos cortes). Incluso, hay ocasiones en que se han encontrado materiales inadecuados en las 


\section{Tecnología en Marcha, \\ Vol. 34, especial. Diciembre 2021 \\ Congreso de Alta Tensión y Aislamiento Electrónico

terminales los cuales causan problemas que inicialmente son prácticamente imperceptibles pero que al paso del tiempo desencadenan fenómenos tales como erosión y arborescencias en aislamientos con fallas catastróficas.

Siempre es necesaria la experiencia, ya que todo resultado requiere siempre de la interpretación de alguien con experiencia. Se habla de sospecha de falla porque ya se debe tener una idea de cómo puede fallar una terminal, es decir, al tener conocimiento de los efectos de campo eléctrico es posible inferir dónde puede existir un defecto y/o posible punto de falla.

La presente propuesta proporciona una técnica adecuada y segura orientada a la anticipada detección de fallas, además, ya que la medición necesaria no requiere contacto con las instalaciones, se puede llevar a cabo en sitio sin interrupción de la energía.

\section{Referencias}

[1] G. J. Paoletti and G. Herman, "Monitoring of electrical equipment failure indicators and zero-planned outages: Past, present and future maintenance practices," 2015 61st IEEE Pulp and Paper Industry Conference (PPIC), 2015, pp. 1-10, doi: 10.1109/PPIC.2015.7165712.

[2] E. Gulski et al., "On-site testing and PD diagnosis of high voltage power cables," in IEEE Transactions on Dielectrics and Electrical Insulation, vol. 15, no. 6, pp. 1691-1700, December 2008, doi: 10.1109/ TDEI.2008.4712673.

[3] P Levesque, P Brémond, J L Lasserre, A Paupert, D L Balageas, Performance of FPA IR cameras and their improvement by time, space and frequency data processing. Part I - Intrinsic characterization of the thermographic system', QIRT Journal, Vol. 2, No 1, pp 97-112, 2005.

[4] G M Carlomagno, P G Berardi, 'Unsteady thermography in non destructive testing', Proceedings 3rd Biannual Information Exchange, St Louis, USA, pp 33-39, 1976.

[5] Condumex Cables, Manual técnico de cables de energía, 4ta ed. México: Grupo Condumex, 2006.

[6] J. Kuffel, \& P. Kuffel, High voltage engineering fundamentals. Newnes. 2000.

[7] R. Arora, \& W. Mosch, High voltage and electrical insulation engineering, John Wiley \& Sons. 2006.

[8] G.G. Raju, Dielectrics in electric fields, CRC press, (2016)

[9] R. Bartnikas, "Detection of partial discharges (Corona) in electrical apparatus," in IEEE Transactions on Electrical Insulation, vol. 25, no. 1, pp. 111-124, Feb. 1990, doi: 10.1109/14.45238.

[10] A. Cavallini, G. C. Montanari and M. Tozzi, "PD apparent charge estimation and calibration: A critical review," in IEEE Transactions on Dielectrics and Electrical Insulation, vol. 17, no. 1, pp. 198-205, February 2010, doi: 10.1109/TDEI.2010.5412018.

[11] Lemke, E., Berlijn, S., Gulski, E., Muhr, H. M., Pultrum, E., Strehl, T., Hauschild, W., Rickmann, J., \& Rizzi, G. (2008). Guide for Electrical Partial Discharge Measurements in compliance to IEC 60270. Electra 241, 60-68.

[12] Young, lan \& Gerbrands, Jan \& Van Vliet, Lucas \& Bibliotheek, Cip-data \& Haag, Den \& Theodore, Young \& Jacob, Gerbrands \& Vliet, Van \& Jozef, Lucas. Fundamentals Of Image Processing, 2004.

[13] R.C.Gonzalez, R.E.Woods, S.L.Eddins, Digital Image Processing Using MATLAB, chap.11, Pearson Prentice Hall, Upper Saddle River, NJ, 2009.

[14] L. Vincent, "Morphological grayscale reconstruction in image analysis: applications and efficient algorithms," in IEEE Transactions on Image Processing, vol. 2, no. 2, pp. 176-201, April 1993, doi: 10.1109/83.217222.

[15] Bozek J., Mustra M., Delac K., Grgic M. (2009) A Survey of Image Processing Algorithms in Digital Mammography. In: Grgic M., Delac K., Ghanbari M. (eds) Recent Advances in Multimedia Signal Processing and Communications. Studies in Computational Intelligence, vol 231. Springer, Berlin, Heidelberg. https://doi. org/10.1007/978-3-642-02900-4_24.

[16] Thierry Pun, A new method for grey-level picture thresholding using the entropy of the histogram, Signal Processing, Volume 2, Issue 3, 1980, Pages 223-237, ISSN 0165-1684, https://doi.org/10.1016/01651684(80)90020-1.

[17] A. Gonzalez-Parada, R. Guzman-Cabrera, M. Torres-Cisneros, \& J. Guzman-Sepulveda, J. R. Comparative Analysis of Thermography Studies and Electrical Measurement of Partial Discharges in Underground Power Cables. International Journal of Thermophysics, 36(9), 2356-2369. 2015 